\title{
GJB1 Gene Analysis in Two Extended Families with X-Linked Charcot-Marie-Tooth Disease
}

\author{
Sabine Kovale ${ }^{a}$ Ruta Terauda $^{a}$ Elina Millere ${ }^{a}$ b Gita Taurina ${ }^{b}$ \\ Daiga Murmane $^{b}$ Jekaterina Isakova ${ }^{a}$ Viktorija Kenina $^{a} b \quad$ Linda Gailite $^{a}$ \\ aScientific Laboratory of Molecular Genetics, Riga Stradins University, Riga, Latvia; \\ ${ }^{b}$ Children's Clinical University Hospital, Riga, Latvia
}

\section{Keywords}

Charcot-Marie-Tooth disease - GJB1 · X-linked Charcot-Marie-Tooth disease

\begin{abstract}
X-linked Charcot-Marie-Tooth (CMT) disease type I (CMTX1) is the second most frequent type of CMT disease caused by pathogenic variants in the GJB1 gene. We described 2 extended cases (families) with CMTX1 with identified pathogenic variants - p.Val139Met and p.Arg215Trp. In both the families, neurological symptoms started earlier in male than in female patients. In some family members, molecular diagnostics was performed prior to neurological investigation due to family cascade screening. There was variable neurological phenotype representing CMT. Conclusions: There is a large clinical heterogeneity in CMTX, even amongst the family members.
\end{abstract}

\section{Introduction}

Charcot-Marie-Tooth disease (CMT) is the most common hereditary sensorimotor neuropathy, with a prevalence of 1:2,500. CMT represents a group of clinically and genetically heterogeneous sensorimotor peripheral neuropathies and is characterized by a wide spectrum of symptoms $[1,2]$. Distal muscle atrophy and weakness, sensory disturbance, foot deformities, such as pes cavus, and absent deep tendon reflexes have been reported from clinically evaluated CMT patients [2,3].X-linked Charcot-Marie-Tooth disease type 1 (CMTX1) (OMIM\# 302800) - caused by defects in the GJB1 gene - is the second most common form of CMT after CMT1A and is the most common CMTX type accounting for 90\% [4]. 
The GJB1 gene encodes the protein connexin 32 (CX32), which is expressed in peripheral nervous system myelinating Schwann cells and thereby in both nerves and ganglia [5-7]. All pathogenic GJB1 variants can be classified into either ones that result in the absence of CX32 protein on the cell membrane and cause cell trafficking defects or ones where CX32 protein is found on the cell membrane but the gating function is changed. In both cases, the CX32 protein has impaired or no function [8]. The loss of CX32 protein function leads to altered ion and small-molecule trafficking and ultimately cell oedema. Furthermore, a gain-of-function defect resulting in central nervous system presentation has been reported [9].

In general, the onset of CMTX1 symptoms is reported during late childhood/adolescence. CMTX occurs earlier and more severely in male individuals; most male patients are clinically symptomatic by the age of $10[9,10]$. For two-thirds of female cases, due to X-inactivation, the mutant allele is expressed in a proportion of the cells, making the symptoms milder [11]. However, one-third of the female patients clinically resemble the affected male ones [12]. Since the longest nerves in the body are affected first, the lower extremities are affected earlier than the upper [3]. Most often, the initial symptom is gait disturbance [10]. Having said that, many individuals experience subtle signs even earlier, such as motor delay, decreased athletic abilities, hip dysplasia, scoliosis, foot abnormalities, or pain; however, these signs can resemble many other childhood neurological diseases $[3,13]$. From the small number of studies on CMTX within families, the phenotypic variance has been noted amongst family members [14-16].

\section{Aim of the Study}

The aim of this study was to examine the phenotype of CMTX in 2 large families where CMTX is caused by 2 pathogenic variants, namely, p.Val139Met located in the transmembrane domain and p.Arg215Trp located in the intracellular domain.

\section{Case Report/Case Presentation}

\section{Materials and Methods}

The study protocol was approved by the Central Medical Ethics Committee of Latvia (No. 3/18-03-21). All participating patients or their parents gave signed informed consent. The patients were from 2 large families where CMTX1 was confirmed by molecular analysis.

DNA was isolated from peripheral blood using a commercial kit (Analytik Jena, Jena, Germany). GJB1 sequencing was performed using Sanger sequencing (BigDye Terminator Kit v.3.1) according to the manufacturer's protocol (Thermo Fisher Scientific, Waltham, MA, USA). The primer sequences used were as previously described (Tomaselli et al. [14] 2017). For probands, all genes were sequenced. DNA sequences were aligned with the reference sequences NG_008357.1, NM_001097642.2, and NP_000157.1. Two variants were identified - p.Arg215Trp (c.643C>T, R215W, and rs879254099) and p.Val139Met (c.415 G>A, V139M, and rs104894812).

For family members, clinical and neurophysiological evaluation was performed in an outpatient setting. Standardised tests used for CMT patients were employed - CMT Neuropathy Score version 2 (CMTNSv2) and 6-min walk distance (6MWD) [17]. Neuropathic pain was evaluated using the DN4 scale, and the patients also responded to an in-house socio-demographic questionnaire. Following the identification of the index patient in both families, targeted screening for the identified variant was performed for family members after genetic consultation. The pedigree and identified genotypes are shown in Figure 1.

Seven members from the family No. 1 (p.Arg215Trp) were tested: 3 female and 4 male individuals. Two of the male patients showed the GJB1 p.Arg215Trp variant in the hemizygous

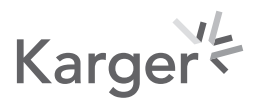


state (ID106 and ID12) (the other 2 male patients were negative), whereas all 3 of the female individuals showed the variant in the heterozygous form (ID11, ID107, and ID105). The aged patients first reported symptoms ranged between 1 year and 14 years. One family member had her molecular diagnosis confirmed within the first year of life (6 months); a nerve conduction study was not performed at the time as examination of her psychomotor development showed it to be appropriate for her age. The other patients were tested because they were already exhibiting a classic symptom of CMTX - pes cavus. The clinical and neurophysiological findings are shown in Table 1. The clinical hereditary neuropathy diagnosis was made for 4 family members and ranged between 7 and 49 years of age. Nerve conduction studies showed axonal polyneuropathy in the majority of the affected family members. Almost all affected family members had a foot deformity. None of the patients had signs of neuropathic pain.

Nine members from family No. 2 (p.Val139Met) were tested: 5 female and 4 male individuals. Two male patients had the GJB1 p.Val139Met variant in the hemizygous state and 4 female patients had the variant in a heterozygous form. The remaining family members $(2$ male and 1 female patients) tested negative for the pathogenic variant. The age at the onset of symptoms ranged between 10 and 17 (average age 13.3) years. Prior to molecular diagnosis, the clinical hereditary neuropathy diagnosis was confirmed for 3 family members ranged between 16 and 41 (average 32) years. Nerve conduction studies showed demyelinating polyneuropathy in half of the family members. Furthermore, pes cavus was observed in 4 of the 6 pathogenic variant-positive family members. CMTNSv2 showed a more severe clinical phenotype in the male patients. These patients also demonstrated the shortest 6MWD, despite not being the oldest family representatives. The youngest family members ( 3 female patients) had the lowest CMTNSv2. The score increased due to nerve conduction study abnormalities only; the normal 6MWD was preserved. Two family members complained about neuropathic pain, indicating possible small nerve fibre damage. None of the CMTX patients exhibited central nervous system involvement, and it had not been previously reported in their medical records.

For the p.Arg215Trp variant, the average age at the onset of first symptoms was 7.5 years for male and 8 years for female patients. For the p.Val139Met variant, it was 11.5 years for male and 17 years for female patients. The family with the p.Arg215Trp variant showed more variable clinical manifestations than the family with the p.Val139Met variant.

\section{Discussion/Conclusion}

Our clinical evaluation of family No. 1 and family No. 2 is consistent with the theoretical information detailed in previous studies [14-16]. The GJB1 gene pathogenic variants p.Val139Met and p.Arg215Trp result in the absence of functional gap junction channels or plaques, although mutant CX32 protein is transported to and inserted into the cell membrane. Both variants have suggested a dominant negative effect in female individuals, with p.Arg215Trp effect being less than p.Val139Met [18]. In our group, male individuals were affected earlier than female ones, that is, opposite to the dominant negative effect.

Fig. 1. Pedigrees with CMTX. Left panel: No. 1 family with the identified p.Arg215Trp variant in the GJB1 gene. Right panel: No. 2 family with the identified p.Val139Met variant in the GJB1 gene. The pedigrees show the ID number and the age of molecular diagnosis (age of clinical symptom manifestation), and identified pathogenic variant and its status. The entries of individuals who are/were affected but have no data are a result of pathogenic variant DNA not being available. NA, not applicable. CMTX, X-linked Charcot-Marie-Tooth disease; ID, identification. 


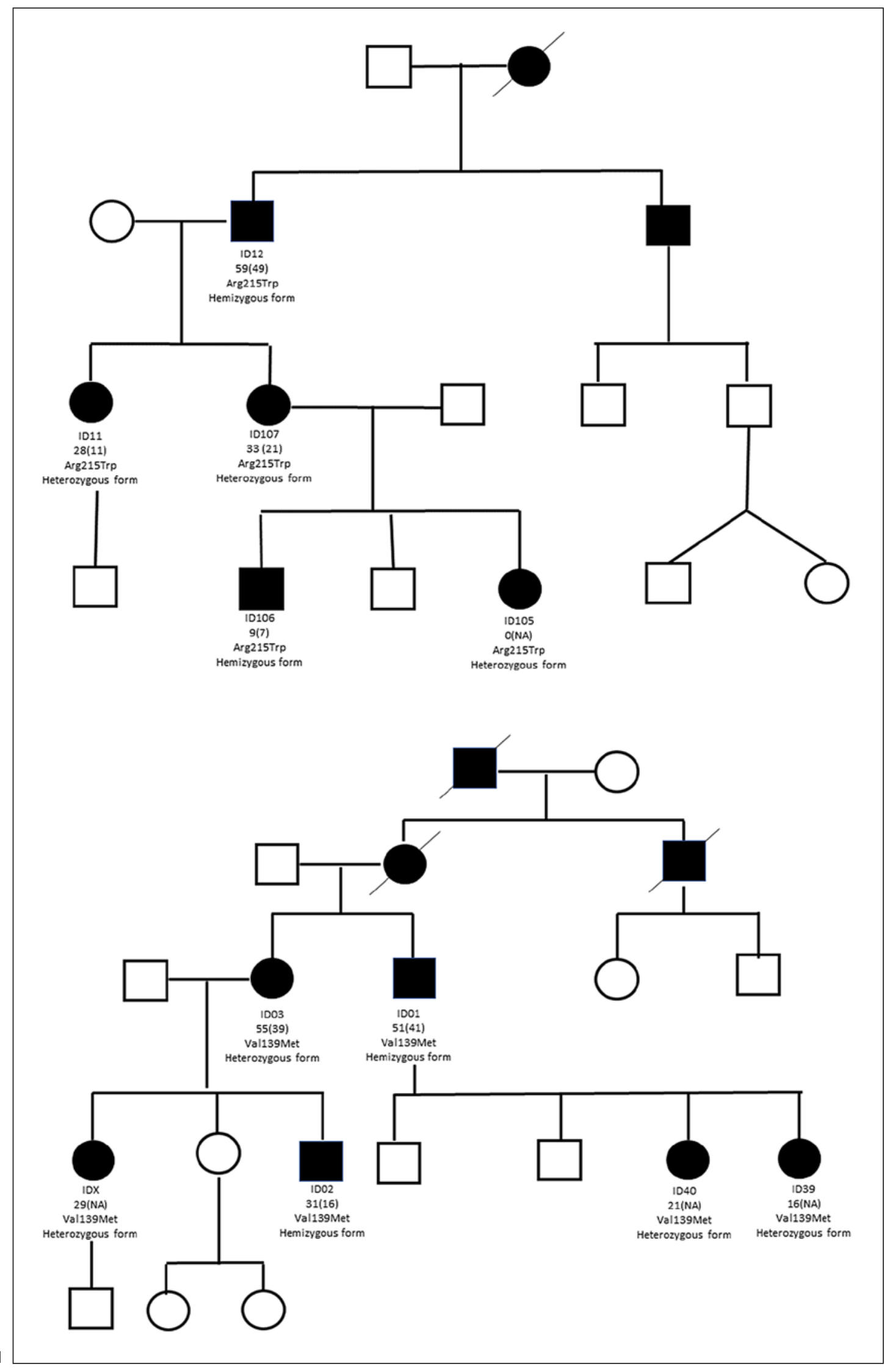

Karger' 


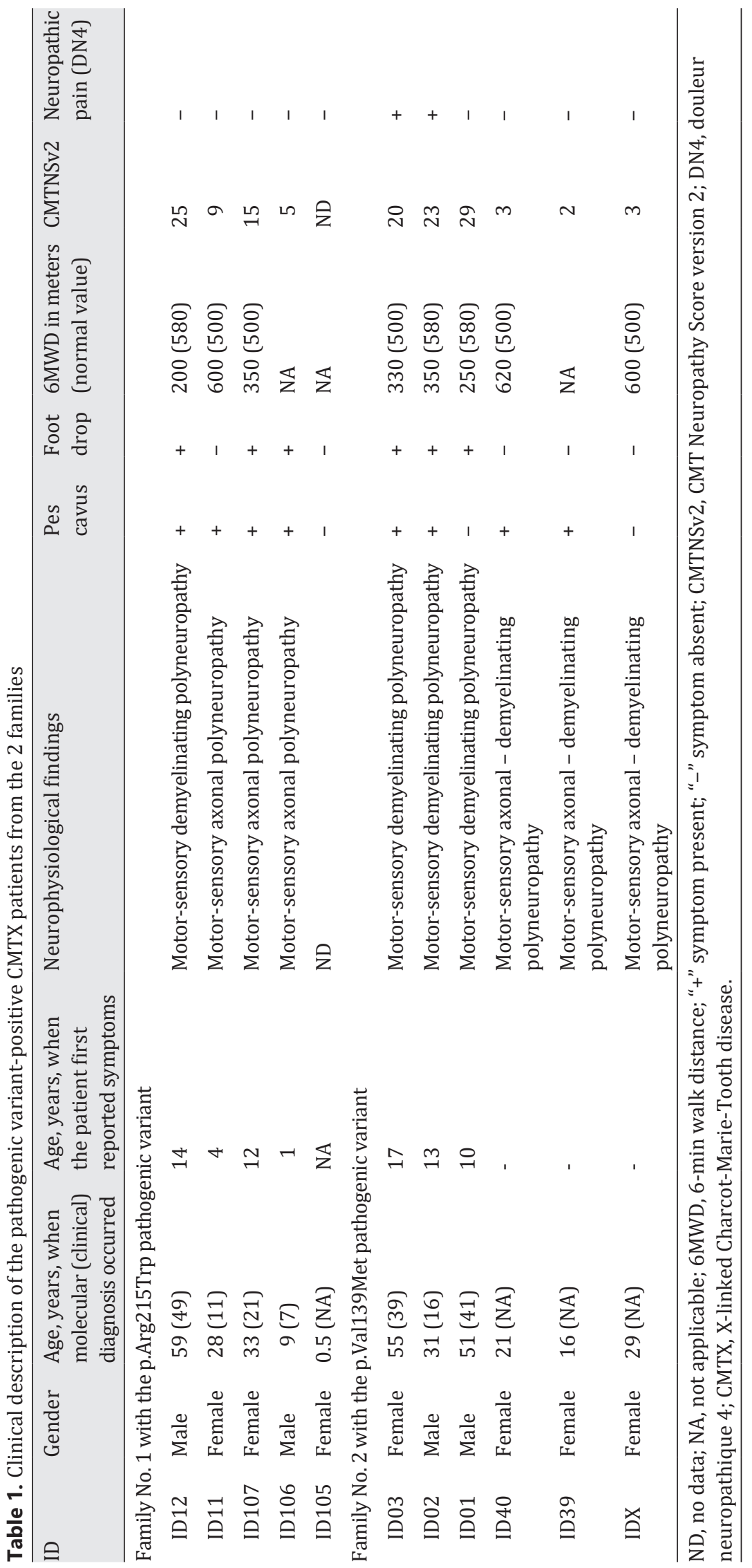


In general, the symptoms of CMTX1 are first reported in the late childhood and earlier in male than in female patients $[4,9]$. Our group of CMTX1 patients followed this trend; the average age at the onset of the first symptoms was 7.75 years for male and 13.3 years for female patients. Furthermore, family No. 1 with the p.Arg215Trp variant exhibited symptoms on average 5.55 years earlier. As the longer nerves are affected first [3], initial symptoms develop in the lower extremities. In line with this, the vast majority of affected individuals in both families (80\%) demonstrated pes cavus and $60 \%$ had foot drop. Indeed, in one of the affected family members, pes cavus was observed at the toddler stage. Karakis et al. [3] suggested that as pes cavus in idiopathic cases is commonly associated with CMT, patients exhibiting this foot deformity should be investigated for this disease. Additionally, Yagerman et al. [13] reported that 78\% of children with bilateral pes cavus were diagnosed with CMT. Regarding the 6MWD results for affected individuals who were unable to achieve the normal value for their gender $(580 \mathrm{~m}$ for me and $500 \mathrm{~m}$ for women [17], it was found that the men performed more poorly than the women.

Other studies have reported central nervous system involvement (both clinical and subclinical) such as acute episodes of motor weakness and dysarthria in CMTX patients [6, 7]. Moreover, magnetic resonance imaging has been used to detect white matter lesions in CMTX patients $[19,20]$. Although the aforementioned approach is beyond the scope of this study, we conclude that our patients did not display nor disclose any episodes or symptoms that would suggest central nervous system involvement.

There are only a few reports in the literature that have studied the correlation between the genotype and phenotype in CMTX patients [2]. We found that the average age when symptoms were first reported was higher in the family with the p.Val139Met variant $(11.5$ years for male and 17 years for female patients) than in the family with the p.Arg $215 \operatorname{Trp}$ variant ( 7.5 years for male and 8 years for female patients). Furthermore, as expected with CMTX being an X-linked disorder, gender differences were observed.

Our findings confirm those of others that there is a large clinical heterogeneity in CMTX, even amongst family members. The 2 families we studied exhibited similar groups of symptoms; however, cold feet and foot callosities, acrocyanosis, and pain were more common in family No. 2 than in family No. 1. Overall, family No. 1 with p.Arg215Trp showed more symptoms than family No. 2 with p.Val139Met.

A limitation of the study is that not all family members, including the affected ones, were available for molecular analysis and neurological evaluation. Also, as the family member consultations stemmed from the index patients, it is likely that some family members were diagnosed based on cascade screening, rather than neurological symptoms.

\section{Statement of Ethics}

Written informed consent was obtained from the patient for publication of this case report and any accompanying images.

\section{Conflict of Interest Statement}

The authors have no conflicts of interest to declare.

\section{Funding Sources}

The study was carried out using the internal research grant in Riga Stradins University.

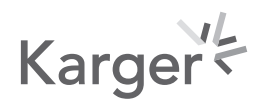




\section{Author Contributions}

S.K. and R.T. prepared the manuscript, performed the experimental part, and analysed the result. G.T. and D.M., as geneticists, were involved in genetic counselling of the patients, collected pedigrees, and reviewed the manuscript. V.K. and E.M., as neurologists, were involved in the clinical examination of the patients and follow-up and reviewed the manuscript. J.I. was involved in the experimental part - preparing Sanger sequencing, and reviewed the manuscript. L.G. was responsible for the result analysis and prepared the manuscript.

\section{References}

1 Skre H. Genetic and clinical aspects of Charcot-Marie-Tooth's disease. Clin Genet. 1974 Aug 1 [cited 2020 Aug 19];6(2):98-118. Available from:

2 Szigeti K, Lupski JR. Charcot-Marie-Tooth disease. Eur J Hum Genet. 2009 Mar 11 [cited 2020 Aug 19];17(6): 703-10. Available from:

3 Karakis I, Gregas M, Darras BT, Kang PB, Royden Jones H. Clinical correlates of charcot-marie-tooth disease in patients with pes cavus deformities. Muscle Nerve [Internet]. 2013 Apr 1 [cited 2020 Sep 1];47(4):488-92. Available from:

4 Wang Y, Yin F. A review of X-linked Charcot-Marie-Tooth disease [Internet]. In: Journal of child neurology. SAGE Publications Inc.; 2016 [cited 2020 Sep 1]. Vol. 31. p. 761-72. Available from:

5 Abrams CK, Scherer SS. Gap junctions in inherited human disorders of the central nervous system [Internet], Biochim Biophys Acta. 2012 [cited 2020 Aug 31]. 1818(8):2030-47. Available from:

6 Halbrich M, Barnes J, Bunge M, Joshi C. A V139M mutation also causes the reversible CNS phenotype in CMTX. Can J Neurol Sci. 2008 [cited 2020 Aug 31];35(3):372-4. Available from:

7 Scherer SS, Deschênes SM, Xu YT, Grinspan JB, Fischbeck KH, Paul DL. Connexin32 is a myelin-related protein in the PNS and CNS. J Neurosci. 1995 Dec 1 [cited 2020 Aug 31];15(12):8281-94. Available from:

8 Mones S, Bordignon B, Fontes M. Connexin 32 is involved in mitosis. Glia. 2012 Mar 1 [cited 2020 Sep 1];60(3): 457-64. Available from:

9 Kleopa KA, Abrams CK, Scherer SS. How do mutations in GJB1 cause X-linked Charcot-Marie-Tooth disease? [Internet]. Brain Res. 2012 [cited 2020 Sep 1];1487:198-205. Available from:

10 Saporta ASD, Sottile SL, Miller LJ, Feely SME, Siskind CE, Shy ME. Charcot-marie-tooth disease subtypes and genetic testing strategies. Ann Neurol. 2011 Jan 1 [cited 2020 Sep 1];69(1):22-33. Available from:

11 Abrams CK, Freidin M. GJB1-associated X-linked Charcot-Marie-Tooth disease, a disorder affecting the central and peripheral nervous systems [Internet]. In: Cell and Tissue Research: Springer Verlag; 2015 [cited 2020 Sep 1]. Vol. 360. p. 659-73. Available from:

12 Siskind CE, Murphy SM, Ovens R, Polke J, Reilly MM, Shy ME. Phenotype expression in women with CMT1X. J Peripher Nerv Syst. 2011 Jun 1 [cited 2020 Sep 1];16(2):102-7. Available from:

13 Yagerman SE, Cross MB, Green DW, Scher DM. Pediatric orthopedic conditions in Charcot-Marie-Tooth disease: a literature review. Curr Opin Pediatr. 2012 Feb [cited 2020 Sep 1];24(1):50-6. Available from:

14 Tomaselli PJ, Rossor AM, Horga A, Jaunmuktane Z, Carr A, Saveri P, et al. Mutations in noncoding regions of GJB1 are a major cause of X-linked CMT. Neurology. 2017 Apr 11 [cited 2020 Sep 1];88(15):1445-53. Available from:

15 Xiao F, Tan JZ, Zhang X, Wang XF. A novel mutation in GJB1 (c.212T >G) in a Chinese family with X-linked Charcot-Marie-Tooth disease. J Clin Neurosci. 2015 Mar 1 [cited 2020 Sep 1];22(3):513-8. Available from:

16 Boerkoel CF, Takashima H, Garcia CA, Olney RK, Johnson J, Berry K, et al Charcot-Marie-Tooth disease and related neuropathies: Mutation distribution and genotype-phenotype correlation. Ann Neurol. 2002 Feb 1 [cited 2020 Sep 1];51(2):190-201. Available from:

17 Crapo RO, Casaburi R, Coates AL, Enright PL, MacIntyre NR, McKay RT, et al. ATS statement: guidelines for the six-minute walk test [Internet]. In: American journal of respiratory and critical care medicine: American Lung Association; 2002 [cited 2020 Sep 1]. Vol. 166. p. 111-7. Available from: http://www.atsjournals.org/doi/ abs/10.1164/ajrccm.166.1.at1102.

18 Omori Y, Mesnil M, Yamasaki H. Connexin 32 mutations from X-linked Charcot-Marie-Tooth disease patients: functional defects and dominant negative effects. Mol Biol Cell. 1996 [cited 2020 Sep 1];7(6):907-16. Available from:

19 Rosser T, Muir J, Panigrahy A, Baldwin EE, Boles RG. Transient leukoencephalopathy associated with X-Linked Charcot-Marie-Tooth disease. J Child Neurol. 2010 May 14;25(8):1013-6. Available from.

20 Al-Mateen M, Craig AK, Chance PF. The central nervous system phenotype of X-Linked Charcot-Marie-Tooth disease: a transient disorder of children and young adults. J Child Neurol. 2014 Feb 11;29(3):342-8. Available from. 